\title{
A Simple Image Processing Approach To ABNORMAL SLiCES DETECTION FROM MRI TUMOR VOLUMES
}

\author{
T.Kalaiselvi, P.Nagaraja and P.Sriramakrishnan \\ Department of Computer Science and Applications, \\ Gandhigram Rural Institute - Deemed University, Gandhigram, Tamil Nadu, India
}

\begin{abstract}
This paper proposed a method for brain tumor detection from the magnetic resonance imaging (MRI) of human head scans. The proposed work explained the tumor detection process by means of image processing transformations and thresholding technique. The MRI images are preprocessed by transformation techniques and thus enhance the tumor region. Then the images are checked for abnormality using fuzzy symmetric measure (FSM). If abnormal, then Otsu's thresholding is used to extract the tumor region. Experiments with the proposed method were done on 17 datasets. Various evaluation parameters were used to validate the proposed method. The predictive accuracy (PA) and dice coefficient (DC) values of proposed method reached maximum.
\end{abstract}

\section{KEYWORDS}

Minima Transform, Thresholding, Transforms, Tumor.

\section{INTRODUCTION}

Brain tumor is cluster of abnormal and an uncontrolled growth of cells in the brain [1]. Brain tumors can be classified according to their origin or degree of aggressiveness. Primary brain tumors arise in the brain, while metastatic brain tumors frequently originate from other parts of the body [2]. Magnetic resonance imaging (MRI) plays important role in many medical imaging applications. MRI provides prosperous information about the human soft tissue anatomy as well as helps to diagnosis of brain tumor [3]. In recent years, MRI has become an important modality for neurological image diagnosis. The combination of different sequences of MRI techniques is used to diagnose tumor [4]. The sequences include T1-weighted, T1-weighted with contrast enhancement (T1c), T2-weighted and fluid attenuated inversion recovery (FLAIR). Nowadays, brain tumor detection for MRI is difficult task for medical applications [5].

In recent years, many approaches have been developed for brain tumor detection. Jayachandran and Dhanasekaran proposed a hybrid algorithm for detection brain tumor in MRI images using statistical features and Fuzzy Support Vector Machine (FSVM) classifier. The proposed technique consists of four stages namely, Noise reduction, Feature extraction, Feature reduction and Classification. Stage I, anisotropic filter is applied for noise reduction and extracting the features. Stage II, obtains the texture features related to MRI images. Stage III, the features of magnetic resonance images have been reduced using principles component analysis to the most essential features. Stage IV, the supervisor classifier based FSVM has been used to classify subjects as normal and abnormal brain MR images [6]. Somasundaram and Kalaiselvi proposed an automatic method to analyze the MRI head scans and detect abnormality in brain due to tumors. This method consist four stages: brain extraction algorithm, transformation, fuzzy segmentation and fuzzy symmetric analysis. This method used two measures: false alarm (FA) 
and missed alarm (MA) to quantify the performance of the method. The mean FA no more detected, however the MA was detecting minimum quantitative value [7]. Logeswari and karnan proposed an enhanced implementation of brain tumor detection using segmentation based on soft computing. The proposed method used two phased for detection tumor. In first phase MRI image of brain is collected. After that using preprocessing technique image is converted into standard form. Second phase for image segmentation using hierarchical self organizing map (HSOM) method is applied on image [8].

Roy and Bandyopadhyay proposed an interactive segmentation method that enables users can quickly and efficiently segment tumors in MRI of brain. In addition to area of the region and edge information the proposed method uses a type of prior information also its detecting the tumor region on exactly from MRI [9]. Padole and Chaudhari proposed an efficient method for brain tumor detection. Combination of two standard algorithm, mean shift and normalized cut is performed to detect the brain tumor surface area in MRI. Segmentation of brain, detects tumor and also its physical dimension and its segmentation accuracy is discussed [10]. Anandgaonkar and Sable proposed a survey on different segmentation techniques applied to MR images for locating tumor. It also includes a proposed method for the same using Fuzzy C-Means algorithm and an algorithm to find area of tumor which is useful to decide type of brain tumor whether it is benign or malignant [11].

The proposed work is an automatic method for extraction of the complete tumor region which overcomes the above said problems and works efficiently for FLAIR and T2-weighted images. The proposed method introduces enhancing process using top and bottom hat transformations and minima transform. Initially MRI head scans are enhanced by using top and bottom-hat process. Extended minima transform is used to separate tumor region from the enhanced image. In resultant segmentation, the fuzzy symmetric measure (FSM) is used to check the abnormality detection. Finally applied Otsu's thresholding in abnormal slice, it extracts the tumor region. The proposed method gives better results for detecting the tumor regions from FLAIR and T2weighted images.

This paper is organized as follows. The image processing transformations are explained in section 2 , the Otsu's thresholding is explained in section 3, the proposed method is explained in section 4, the results and discussion are given in section 5 and the conclusion is given in section 6 .

\section{Image Processing Transformations}

\subsection{Top-hat and Bottom-hat Transforms}

The top-hat transform is defined as the difference between the input image and its opening by some structuring element [12]. Structuring element (SE) of proposed method is given by,

$$
\mathrm{SE}=(\text { 'disk’, 15) }
$$

The application of these transforms is in removing objects from an image by using an SE in the opening and closing that does not fit the objects to be removed. The difference then yields an image with only the removed objects.

Then, the top-hat transform of $f$ is given by:

$$
T_{w}(f)=f-\left(f^{\circ} h\right)
$$


where $\circ$ denotes the opening operation. The top-hat transform is an operation that extracts small elements and details from given images. The top-hat transform performs light objects on a dark background.

The bottom-hat transform is defined dually as the difference between the closing and the input image. Then, the bottom-hat transform of $f$ is given by:

$$
T_{b}(f)=(f[b)-f
$$

where $\bullet$ is the closing operation. Bottom-hat performs morphological bottom-hat filtering on the grayscale or binary input image. The bottom-hat performs for dark objects on a light background.

\subsection{Extended Minima Transform}

The extended minima transform, which is the regional minima of the H-minima transform. Extended minima used 8-connected neighborhoods for 2-D images and 26-connected neighborhoods for 3-D image. Performing the $H$-minima transform on the inverse distance image can effectively decrease over segmentation is reduced to some extend after applying morphological filters. The $H$-minima transform [13] is performed by:

$$
H_{h}(f)=R_{f}(f+h)
$$

where $h$ represents the given depth. $R$ represents the reconstruction and erosion operators, respectively.

\section{Otsu's Method}

This method is called as optimum threshold method and provides satisfied results in MRI brain images [14]. Otsu's thresholding involves all possible threshold values and calculate the pixel levels in each side of the threshold. This threshold value separates the foreground or background of pixels. This algorithm compute the image to be threshold contains the two classes of pixels. We can use the within class variance, it is the weighted sum of the variances of each foreground and background [15].

$$
\sigma_{\text {within }}^{2}(T)=W_{B}(T) \sigma_{b g}^{2}(T)+W_{F}(T) \sigma_{f g}^{2}(T)
$$

Where,

$$
W_{B}(T)=\sum_{i=0}^{T=1} p(i) \quad W_{F}(T)=\sum_{i=T}^{N-1} p(i)
$$

$p_{\mathrm{i}}-$ is the probability of occurring of pixel value $\mathrm{x}_{\mathrm{i}}$

The mean of foreground and background pixels is,

$$
\mu_{B}(T)=\frac{1}{W_{B}(T)} \sum_{i=1}^{T} i P(i)
$$


The International Journal of Multimedia \& Its Applications (IJMA) Vol.8, No.1, February 2016

$$
\mu_{F}(T)=\frac{1}{W_{F}(T)} \sum_{i=1}^{T} i P(i)
$$

The variance of foreground and background pixels is,

$$
\begin{aligned}
\sigma_{b g}^{2}(T) & =\frac{1}{W_{B}(T)} \sum_{i=1}^{T}\left(i-\mu_{B}(T)\right)^{2} P(i) \\
\sigma_{f g}^{2}(T) & =\frac{1}{W_{F}(T)} \sum_{i=1}^{T}\left(i-\mu_{F}(T)\right)^{2} P(i)
\end{aligned}
$$

$\sigma_{b g}^{2}(T)$-variance of the pixels in the background.

$\sigma_{f g}^{2}(T)$-variance of the pixels in the foreground.

\section{Proposed Method}

This proposed method is a fully automated brain tumor detection method. The enhanced MRI images are produced by top, bottom-hat, minima transformations and segmented by Otsu's thresholding to extract the tumor portion from both T2-weighted and FLAIR images. The flow chart of tumor extraction methods are shown in Figure 1.

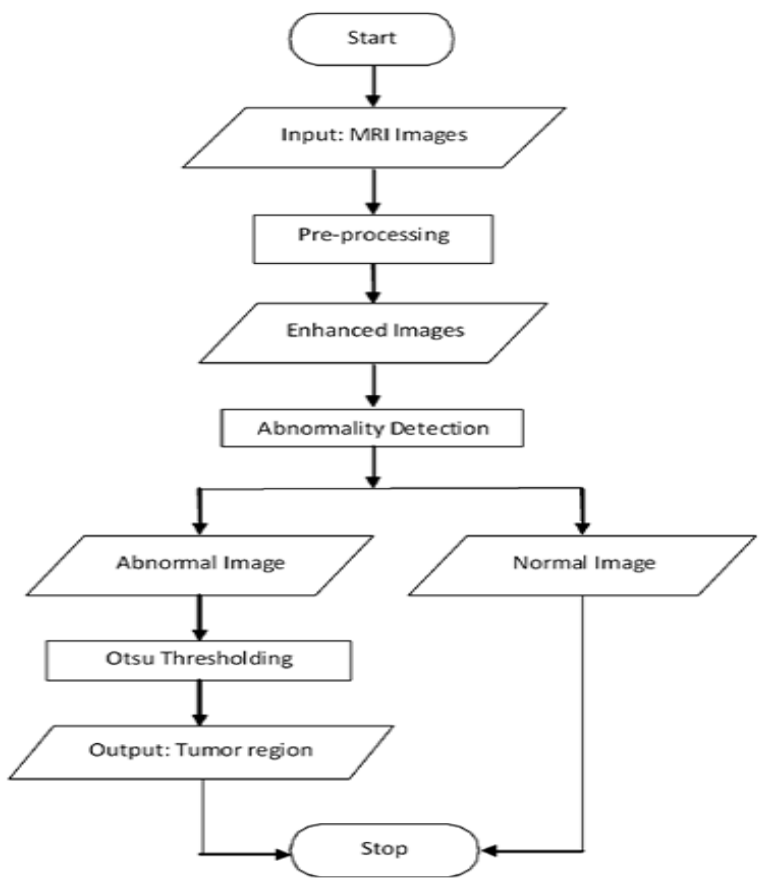

Figure 1. Flow chart for proposed method 


\subsection{Pre-processing}

The proposed method initially has done a pre-processing technique on MRI images. The flow diagram of preprocessing method is given in Figure 2.

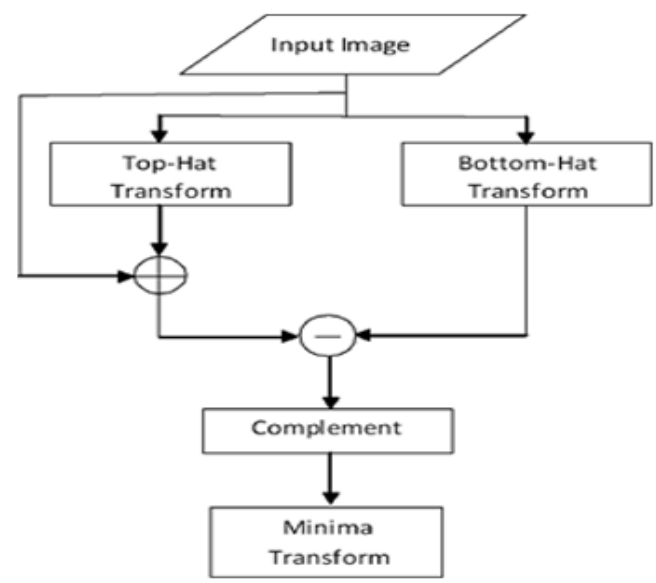

Figure 2. Flow chart for preprocessing

The datasets are processed by top-hat and bottom-hat transformation for enhancing the original image. The preprocessing images are shown in Figure 2. The original image is shown in column 1. The top-hat transformation as shown in column 2 is used to maximize the contrast between the objects and the gaps that separate them from each other. The top-hat transform is defined dually as the difference between the opening and the input image. Bottom-hat performs morphological bottom-hat filtering on the grayscale or binary input image and performs dark objects on a light background. The bottom-hat transform is defined dually as the difference between the closing and the input image. The bottom-hat transform image is shown in column 3. Then add the top-hat image to the original image, and then subtracts the bottom-hat image. This stage tumor area get sharpened region and it is shown in column 4

Then complement the function is used to enhance the image based on the intensity valleys as represented in column 5 . The minima transformation is used to detect the intensity valleys deeper than a particular threshold with that function. It removes local peaks which are lower than $h$ intensity values from the background. Based on the analysis done during our experiments, $\mathrm{h}$ is set to 8 . The enhanced tumor regions extracted by using extended minima transform is shown in column 6 . In column 6 , the normal volume enhanced images are given in row 1.
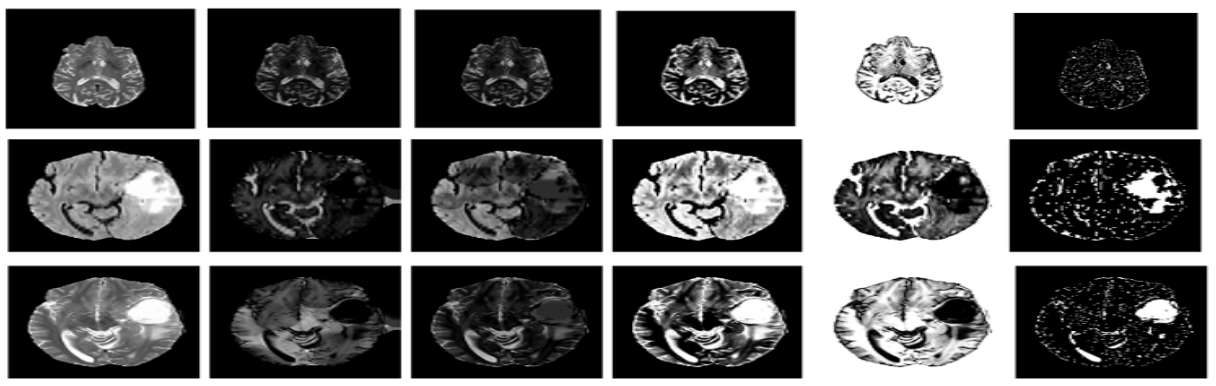

Figure 3. Preprocessing Stage, original images for Normal, FLAIR and T2-weighted 
Figure 3. Preprocessing Stage, original images for Normal, FLAIR and T2-weighted are in column 1, top-hat images are in column 2, bottom-hat images are in column 3, enhanced images are in column 4, complement images are in column 5 and minima transformation images are in column 6.

\subsection{Abnormality Detection}

Abnormal tissues in the enhanced tumor region image can be detected by measuring the symmetry of enhanced tumor image. Here the symmetry is computed by the fuzzy symmetric measure (FSM) [16] given by,

$$
F 5 M=\frac{1}{1+\left(\frac{n_{L}-n_{B}}{2}\right)^{2}}
$$

where $n_{L}$ and $n_{R}$ are the number of foreground (white) pixels in the left and right half of the given image present at either side of the central vertical line of slice. The symmetry values calculated from normal enhanced image are generally much larger than 0.1 , and the values for abnormal enhanced image are much smaller than 0.1 [7] [17].

\subsection{Brain Tumor Detection}

Then the process is carried out by abnormal slice. The tumor extraction method is used by Otsu's thresholding technique. It separates the tumor region from background. In the resultant detection, tumors are extract from the abnormal slice and as a largest connected region as shown in Figure 4.

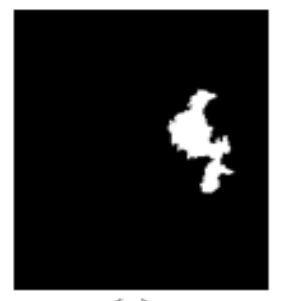

(a)

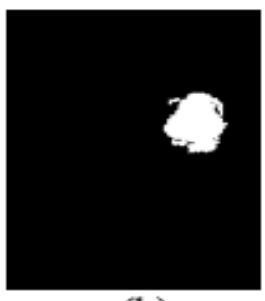

(b)

Figure 4. (a) FLAIR tumor region, (b) T2-weighted tumor region.

\section{RESUlTS AND DiscuSSION}

Our algorithm was implemented in MATLAB2009 on a PC with Intel Pentium Core Duo 1.6GHz processor and 512MB RAM. Our proposed method used 17 datasets from Brain Tumor Image Repository (BTIR) maintained by our research group [18]. The first two datasets (N01-N02) consisting of 2 normal volumes and V01-V15 are abnormal volumes with glioma brain tumor. In tumor portion, two types of outputs from FLAIR and T2-weighted were added and its represents to the complete tumor region is as shown in Figure 5.The qualitative validation in the form of visual inspection is done with some of the sample MRI brain images shown in Figure 6. In Figure 6 , the original FLAIR images are given in column 1, the original T2-weighted MRI images are given in column 2, the corresponding ground truth images are given in column 3 , and the results of proposed method are given in column 4. This proposed work gives good results for glioma images. 


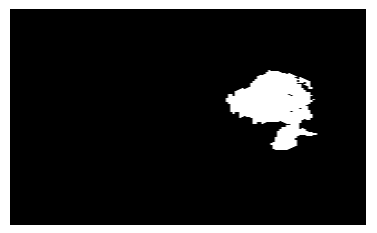

Figure 5. Complete tumor region.

For quantitative validation, the performance is checked against three parameters. They are predictive accuracy (PA), dice coefficient (DC). The segmented images could have an error rate and an instance may either fail to identify an abnormality or identify an abnormality when there is none. The parameter PA is used to describe the error rate by the terms true and false positive (TP, FP) and true and false negative (TN, FN).

$$
P A(\%)=\frac{T P+T N}{T P+T N+F P+F N}=100
$$

where,

$\mathrm{TP}=$ the test result is positive in the abnormal cases correctly classified.

$\mathrm{TN}=$ the test result is negative in the normal cases correctly classified.

$\mathrm{FP}=$ the test result is positive in the normal cases classified abnormal.

$\mathrm{FN}=$ the test result is negative in the abnormal cases classified normal.

The parameter DC is used to compare the similarity between manual segmentation and the result of the proposed work. The value for DC ranges from 0 to 1 where 0 for no agreement and 1 for exact agreement.

The DC is given by:

$$
D C(A, B)=\frac{2|A \cap B|}{|A|+|B|}
$$

where A represents the ground truth image and B represents the proposed result image.

Table 1 is shown the abnormal detection by evaluated parameters FA and MA. The missed alarm is $2.3 \%$ from the proposed method and no more FA in entire datasets. The performance analysis of proposed method is based on the parameters PA and DC values given in Table 2. The graph shows the quantitative representation of PA and DC of proposed method with line representation. In Figure 7, $\mathrm{X}$ axis represents volume id considered for experiment and $\mathrm{Y}$ axis represents the PA and DC values proposed method. The PA and DC of proposed method are $98 \%$ and $75 \%$ in glioma images. Our proposed method gives good results for glioma images.
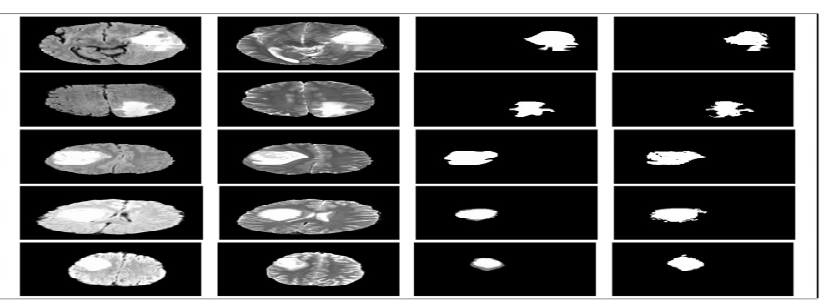

Figure 6. FLAIR images are in column 1, T2-weighted images are in column 2, corresponding ground truth images are in column 3 and the results of proposed method are in column 4.

Table 1. FA and MA values for proposed method 
The International Journal of Multimedia \& Its Applications (IJMA) Vol.8, No.1, February 2016

\begin{tabular}{|l|l|l|l|l|l|l|l|l|}
\hline \multirow{2}{*}{ No } & \multirow{2}{*}{$\begin{array}{c}\text { Vol. } \\
\text { id }\end{array}$} & $\begin{array}{c}\text { Tota } \\
\text { Slice }\end{array}$ & \multicolumn{1}{|c|}{ Abnormal Slice } & \multicolumn{2}{|c|}{ FA } & \multicolumn{2}{|c|}{ MA } \\
\cline { 3 - 9 } & & Actual & Detected & Slices & \% & \multicolumn{2}{|c|}{ Slices } & $\%$ \\
\hline 1 & N01 & 54 & - & - & 0 & 0 & 0 & 0 \\
\hline- & - & 43 & - & - & 0 & 0 & 0 & 0 \\
\hline 3 & V01 & 176 & $46-119$ & $50-119$ & 0 & 0 & $46-49$ & 2 \\
\hline 4 & V02 & 176 & $44-110$ & $47-106$ & 0 & 0 & $44-46,107-110$ & 4 \\
\hline 5 & V03 & 176 & $62-146$ & $67-141$ & 0 & 0 & $62-66,142-146$ & 6 \\
\hline 6 & V04 & 176 & $81-150$ & $81-145$ & 0 & 0 & $146-150$ & 3 \\
\hline 7 & V05 & 176 & $68-131$ & $69-131$ & 0 & 0 & 68 & 0.6 \\
\hline 8 & V06 & 176 & $87-161$ & $87-160$ & 0 & 0 & 161 & 0.6 \\
\hline 9 & V07 & 176 & $78-137$ & $81-135$ & 0 & 0 & $78-80,136,137$ & 3 \\
\hline 10 & V08 & 176 & $55-133$ & $60-133$ & 0 & 0 & $55-59$ & 3 \\
\hline 11 & V09 & 176 & $75-147$ & $77-145$ & 0 & 0 & $75,76,146,147$ & 2 \\
\hline 12 & V10 & 176 & $77-117$ & $80-115$ & 0 & 0 & $77-79,116,117$ & 3 \\
\hline 13 & V11 & 230 & $118-167$ & $118-166$ & 0 & 0 & 167 & 0.6 \\
\hline 14 & V12 & 165 & $78-119$ & $78-119$ & 0 & 0 & 0 & 0.4 \\
\hline 15 & V13 & 220 & $81-132$ & $85-127$ & 0 & 0 & $81-84,127-132$ & 5 \\
\hline 16 & V14 & 230 & $94-120$ & $96-114$ & 0 & 0 & $95-96,115-120$ & 2 \\
\hline 17 & V15 & 163 & $61-101$ & $61-101$ & 0 & 0 & 0 & 0 \\
\hline & Average Performance & & $\mathbf{0}$ & $\mathbf{0}$ & & $\mathbf{2 . 3}$ \\
\hline
\end{tabular}

Table 2. PA and DC values of the proposed method

\begin{tabular}{|c|l|c|c|}
\hline S.No & Vol. id & $\begin{array}{c}\text { PA (\%) } \\
\text { (Complete } \\
\text { Tumor) }\end{array}$ & $\begin{array}{c}\text { DC (\%) } \\
\text { (Complete } \\
\text { Tumor) }\end{array}$ \\
\hline 1 & V01 & 98 & 84 \\
\hline 2 & V02 & 97 & 73 \\
\hline 3 & V03 & 97 & 87 \\
\hline 4 & V04 & 98 & 85 \\
\hline 5 & V05 & 98 & 60 \\
\hline 6 & V06 & 98 & 77 \\
\hline 7 & V07 & 98 & 62 \\
\hline 8 & V08 & 98 & 81 \\
\hline 9 & V09 & 96 & 69 \\
\hline 10 & V10 & 99 & 61 \\
\hline 11 & V11 & 99 & 92 \\
\hline 12 & V12 & 97 & 75 \\
\hline 13 & V13 & 98 & 73 \\
\hline 14 & V14 & 98 & 74 \\
\hline 15 & V15 & 99 & 77 \\
\hline & Mean & $\mathbf{9 7 . 9}$ & $\mathbf{7 5 . 3}$ \\
\hline & & & \\
\hline
\end{tabular}




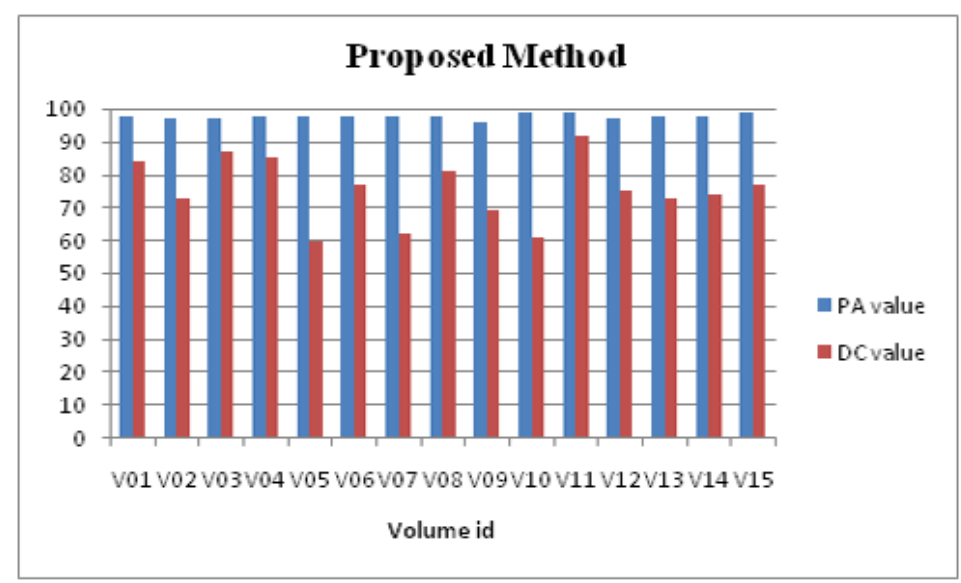

Figure 7. PA and DC measurement Graph for the proposed method

\section{Conclusions}

The proposed work developed a brain tumor detection method using top-hat, bottom-hat, minima transformations and Otsu's thresholding. The experimental method is done on 17 datasets. This work detected the abnormal portion of the brain which is present in MRI images. The advantage of this proposed method is it takes minimum missed alarm while detecting the tumor in MRI images. It gives $75 \%$ similarity index while compared with ground truth dataset.

\section{REFERENCES}

[1] Somasundaram, K. \& Kalaiselvi, T., (2010) "Automatic detection of brain tumor from MRI scans using maxima transform”, National Conference on Image Processing (NCIMP), pp136-141.

[2] Kalaiselvi, T. \& Nagaraja, P., (2015) "Brain Tumor Segmentation of MRI Brain Images through FCM clustering and Seeded Region Growing Technique”, International Journal of Applied Engineering Research, vol.10, no.76, pp427-432.

[3] Kalaiselvi, T., (2011) Brain Portion Extraction and Brain Abnormality Detection from Magnetic Resonance Imaging of Human Head Scans, Pallavi Publications.

[4] Drevelegas, A. \& Papanikolaou, (2011) "Imaging modalities in brain tumors", Imaging of Brain Tumors with Histological Correlations, chapter 2, pp 13-34.

[5] Kalaiselvi, T. \& Nagaraja, P., (2015) "A Rapid Automatic Brain Tumor Detection Method for MRI Images using Modified Minimum Error Thresholding Technique" International Journal of Imaging Systems and Technology, Vol.25, No.1, pp77-85.

[6] Jayachandran, A. \& Dhanasekaran, R., (2013) "Brain Tumor Detection and Classification of MR Images Using Texture Features and Fuzzy SVM Classifier", Research Journal of Applied Sciences, Engineering and Technology, Vol. 6, No.12, pp2264-2269.

[7] Somasundaram, K. \& Kalaiselvi, T., (2010) "Fully Automatic Method to Identify Abnormal MRI Head Scans using Fuzzy Segmentation and Fuzzy Symmetric Measure”, ICGST International Journal on Graphics, Vision and Image Processing (GVIP), Vol.10, No.3, pp1-9.

[8] Logeswari, T. \& Karnan, M., (2010)"An Enhanced Implementation of Brain Tumor Detection Using Segmentation Based on Soft Computing", International Conference on Signal Acquisition and Processing

[9] Roy, S. \& Bandyopadhyay S.K., (2012) "Detection and Quantification of Brain Tumor from MRI of Brain and its Symmetric Analysis", International Journal of Information and Communication Technology Research, Vol.22.

[10] Padole, V.B \& Chaudhari, D.S., (2012) "Detection of Brain Tumor in MRI Images Using Mean Shift Algorithm and Normalized Cut Method", International Journal of Engineering and Advanced Technology. 
[11] Anandgaonkar, G.P., and Sable, G.S., "Detection and Identification of Brain Tumor in Brain MR Images Using Fuzzy C-Means Segmentation", International Journal of Advanced Research in Computer and Communication Engineering, vol.2, no.10, 2013, pp-3964-3967.

[12] Thapar, S. \& Garg, S., (2012) "Study and Implementation of Various Morphology Based Image Contrast Enhancement Techniques", International Journal of Computing Business Research, pp22296166.

[13] Jung, C. \& Kim, C., (2010) "Segmenting Clustered Nuclei Using H-minima Transform-Based Marker Extraction and Contour Parameterization”, IEEE Transactions on Biomedical Engineering, Vol.57, No.10, pp2600-2604.

[14] Kalaiselvi, T. \& Nagaraja. P., (2013), "A Robust Thresholding Technique for Image Segmentation from Gray Images." in Proceedings of the International Conference on Applied Mathematics and Theoretical Computer Science, p. 183-188.

[15] Otsu, N., (1979) "A Threshold Selection from Gray level Histograms," IEEE Transactions of systems, Man and Cybernetics (SMC), Vol. 9, No.1, pp62-66.

[16] Zimmermann, H. J., (1991) Fuzzy Set Theory and its Applications, Kluwer Academic Publishers, second edition, Boston, MA.

[17] Clark, M.C., Hall, L.O., Goldgof, D.B., Velthuizen, R., Murtagh, F.R. \& Silbiger, M.S., (1998) "Automatic Tumor Segmentation using Knowledge-Based Technique", IEEE Transactions on Medical Imaging, Vol. 17, No. 2, pp187-201.

[18] Kalaiselvi, T., "Brain Tumor Image Repository (BTIR)", Department of Computer Science and Applications, Gandhigram Rural Institute- Deemed University, Gandhigram.

\section{Authors}

Kalaiselvi T. currently working as an Assistant Professor in Department of Computer Science and Applications, Gandhigram Rural Institute - Deemed University, Dindigul, India. She received her Bachelor of Science (BSc) degree in Mathematics and Physics in the year 1994 and Master of Computer Applications (MCA) degree in the year 1997 from Avinashilingem University, Coimbatore. She received her Ph.D (Full-time) degree from Gandhigram Rural Institute in the year 2010. In the year 2008, she received a project from Department of Science and

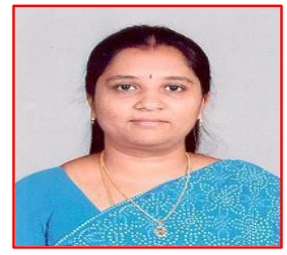
Technology (DST), Government of India under Scheme for Young Scientists and Professionals (SYSP) by Science for Equity, Empowerment and Development (SEED) Division for three years (2008-2011). Her research focuses on Brain Image Processing and brain tumor or lesion detection from MR Head Scans to enrich the Computer Aided Diagnostic process, Telemedicine and Tele radiology services. She is Academic Community Member (ACM) in International Congress for Global Science and Technology (ICGST), Life Member (LM) in Indian Society for Technical Education (ISTE) and Lifetime Member (LM) in Telemedicine Society of India (TSI).

Nagaraja P. is a Research Scholar (Full-time) in the Department of Computer Science and Applications, Gandhigram Rural Institute - Deemed University, Dindigul, India. He received his Bachelor of Science (B.Sc) degree in Physics in the year 2008 and Master of Computer Applications (MCA) degree in the year 2011 from Gandhigram Rural Institute - Deemed University. He is currently pursuing Ph.D. degree in Gandhigram Rural Institute- Deemed University. His research focuses on Brain Tissue Segmentation in MRI Head scans.

P. Sriramakrishnan received his Bachelor of Science (B.Sc.) degree in 2011 from Bharathidasan University, Trichy, Tamilnadu, India. He received Master of Computer Application (M.C.A) degree in 2014 from Gandhigram Rural InstituteDeemed University, Dindigul, Tamilnadu, India. He worked as Software Developer in Dhvani Research and Development Pvt. Ltd, Indian Institute of Technology Madras Research Park, Chennai during May 2014 - March 2015. He is currently

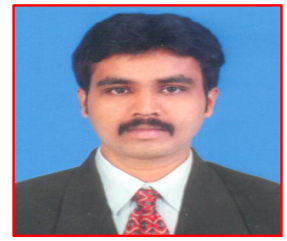
pursuing Ph.D. degree in Gandhigram Rural Institute- Deemed University. His research focuses on Medical Image Processing and Parallel Computing. He has qualified UGC-NET for Lectureship in June 2015. 